\title{
A CLASS OF CONSERVATIVE FOUR-DIMENSIONAL MATRICES
}

\author{
CELAL ÇAKAN AND BILAL ALTAY
}

Received 5 October 2005; Accepted 2 July 2006

The concepts $P$ - lim sup and $P$ - lim inf for double sequences were introduced by Patterson in 1999. In this paper, we have studied some new inequalities related to these concepts by using the $\mathrm{RH}$-conservative four-dimensional matrices.

Copyright (C 2006 C. Çakan and B. Altay. This is an open access article distributed under the Creative Commons Attribution License, which permits unrestricted use, distribution, and reproduction in any medium, provided the original work is properly cited.

\section{Introduction}

A double sequence $x=\left[x_{j k}\right]_{j, k=0}^{\infty}$ is said to be convergent to a number $l$ in the Pringsheim sense or $P$-convergent if for every $\varepsilon>0$, there exists $N \in \mathbb{N}$, the set of natural numbers, such that $\left|x_{j k}-l\right|<\varepsilon$ whenever $j, k>N$, [5]. In this case, we write $P-\lim x=l$. In what follows, we will write $\left[x_{j k}\right]$ in place of $\left[x_{j k}\right]_{j, k=0}^{\infty}$.

A double sequence $x$ is said to be bounded if there exists a positive number $M$ such that $\left|x_{j k}\right|<M$ for all $j, k$, that is, if

$$
\|x\|=\sup _{j, k}\left|x_{j k}\right|<\infty .
$$

Let $\ell_{\infty}^{2}$ be the space of all real bounded double sequences. We should note that in contrast to the case for single sequences, a convergent double sequence need not be bounded. By $c_{2}^{\infty}$, we mean the space of all $P$-convergent and bounded double sequences.

Let $A=\left[a_{j k}^{m n}\right]_{j, k=0}^{\infty}$ be a four-dimensional infinite matrix of real numbers for all $m, n=$ $0,1, \ldots$ The sums

$$
y_{m n}=\sum_{j=0}^{\infty} \sum_{k=0}^{\infty} a_{j k}^{m n} x_{j k}
$$

are called the $A$-transforms of the double sequence $x$. We say that a sequence $x$ is $A$ summable to the limit $s$ if the $A$-transform of $x$ exists for all $m, n=0,1, \ldots$ and convergent 
2 A class of conservative four-dimensional matrices

in the Pringsheim sense, that is,

$$
\begin{gathered}
\lim _{p, q \rightarrow \infty} \sum_{j=0}^{p} \sum_{k=0}^{q} a_{j k}^{m n} x_{j k}=y_{m n}, \\
\lim _{m, n \rightarrow \infty} y_{m n}=s .
\end{gathered}
$$

A matrix $A=\left[a_{j k}^{m n}\right]$ is said to be RH-regular (see $\left.[1,6]\right)$ if $A x \in c_{2}^{\infty}$ and $P-\lim A x=$ $P-\lim x$ for each $x \in c_{2}^{\infty}$. If a matrix $A$ is RH-regular, then we write $A \in\left(c_{2}^{\infty}, c_{2}^{\infty}\right)_{\text {reg. It is }}$ shown that $A$ is RH-regular if and only if

$$
\begin{gathered}
P-\lim _{m, n} a_{j k}^{m n}=0 \quad \text { for each } j, k, \\
P-\lim _{m, n} \sum_{j} \sum_{k} a_{j k}^{m n}=1, \\
P-\lim _{m, n} \sum_{j}\left|a_{j k}^{m n}\right|=0 \quad \text { for each } k, \\
P-\lim _{m, n} \sum_{k}\left|a_{j k}^{m n}\right|=0 \quad \text { for each } j, \\
\|A\|=\sup _{m, n} \sum_{j} \sum_{k}\left|a_{j k}^{m n}\right|<\infty .
\end{gathered}
$$

A matrix $A=\left[a_{j k}^{m n}\right]$ is said to be RH-conservative if $A x \in c_{2}^{\infty}$ for each $x \in c_{2}^{\infty}$. In this case, we write $A \in\left(c_{2}^{\infty}, c_{2}^{\infty}\right)$. One can prove that $A$ is $\mathrm{RH}$-conservative if and only if the condition (1.8) holds and

$$
\begin{gathered}
P-\lim _{m, n} a_{j k}^{m n}=v_{j k} \quad \text { for each } j, k, \\
P-\lim _{m, n} \sum_{j} \sum_{k} a_{j k}^{m n}=v \quad \text { exists, } \\
P-\lim _{m, n} \sum_{j}\left|a_{j k}^{m n}-v_{k l}\right|=0 \quad \text { for each } k, \\
P-\lim _{m, n} \sum_{k}\left|a_{j k}^{m n}-v_{k l}\right|=0 \quad \text { for each } k .
\end{gathered}
$$

For an $\mathrm{RH}$-conservative matrix $A$, we can define the functional

$$
\Gamma(A)=v-\sum_{j} \sum_{k} v_{j k}
$$

where $\sum_{j} \sum_{k}\left|v_{j k}\right|<\infty$ which follows from (1.8) and (1.9). Note that $\Gamma(A)=1$, when $A$ is an RH-regular matrix.

Móricz and Rhoades [2] have defined almost convergence of a double sequence as follows. 
A double sequence $x=\left[x_{j k}\right]$ of real numbers is said to be almost convergent to a limit $l$ if

$$
\lim _{p, q \rightarrow \infty} \sup _{m, n \geq 0}\left|\frac{1}{p q} \sum_{j=m}^{m+p-1} \sum_{k=n}^{n+q-1} x_{j k}-l\right|=0 \quad \text { uniformly in } m, n=1,2, \ldots
$$

Note that a convergent single sequence is also almost convergent but for a double sequence this is not the case, that is, a convergent double sequence need not be almost convergent. However, every bounded convergent double sequence is almost convergent. By $f_{2}$ we denote the space of all almost convergent double sequences. A matrix $A \in\left(f_{2}, c_{2}^{\infty}\right)_{\text {reg }}$ is said to be strongly regular and the conditions of strong regularity are known [2].

For any real bounded double sequence $x$, the concepts $l(x)=P-\liminf x$ and $L(x)=$ $P$ - limsup $x$ have been introduced in [4] and also an inequality related to the $P-\limsup$ has been studied as follows.

Lemma 1.1 [4, Theorem 3.2]. For any real double sequence $x, P-\lim \sup A x \leq P-$ limsup $x$ if and only if $A$ is RH-regular and

$$
P-\lim _{m, n} \sum_{j} \sum_{k}\left|a_{j k}^{m n}\right|=1
$$

Let us define the sublinear functionals $L^{\text {ast }}(x), l^{\text {ast }}(x)$ on $\ell_{\infty}^{2}$ as follows:

$$
\begin{aligned}
& L^{\text {ast }}(x)=P-\limsup _{p, q \rightarrow \infty} \sup _{m, n \geq 0} \frac{1}{p q} \sum_{j=m}^{m+p-1} \sum_{k=n}^{n+q-1} x_{j k}, \\
& l^{\text {ast }}(x)=P-\liminf _{p, q \rightarrow \infty} \sup _{m, n \geq 0} \frac{1}{p q} \sum_{j=m}^{m+p-1} \sum_{k=n}^{n+q-1} x_{j k} .
\end{aligned}
$$

Then, the MR-core of a real bounded double sequence $x$ is the closed interval $\left[l^{\text {ast }}(x)\right.$, $L^{\text {ast }}(x)$ ], [3]. Also, it is proved in [3] that $L(A x) \leq L^{\text {ast }}(x)$ for all $x \in \ell_{\infty}^{2}$ if and only if $A$ is strongly regular and (1.15) holds.

In this paper, we have proved some new inequalities related to the $P-\lim$ sup by using the RH-conservative matrices.

\section{The main results}

Firstly, we need two lemmas, the first can be obtained from [4, Lemma 3.1].

Lemma 2.1. If $A=\left[a_{j k}^{m n}\right]$ is a matrix such that the conditions (1.4), (1.6), (1.7), and (1.8) hold, then for any $y \in \ell_{\infty}^{2}$ with $\|y\| \leq 1$,

$$
P-\limsup \sum_{j, n} \sum_{k} a_{j k}^{m n} y_{j k}=P-\limsup _{m, n} \sum_{j} \sum_{k}\left|a_{j k}^{m n}\right| .
$$


4 A class of conservative four-dimensional matrices

Lemma 2.2. Let $A=\left[a_{j k}^{m n}\right]$ be $R H$-conservative and $\lambda \in \mathbb{R}^{+}$. Then,

$$
P-\limsup \sum_{m, n} \sum_{k}\left|a_{j k}^{m n}-v_{j k}\right| \leq \lambda
$$

if and only if

$$
\begin{aligned}
& P-\limsup _{m, n} \sum_{j} \sum_{k}\left(a_{j k}^{m n}-v_{j k}\right)^{+} \leq \frac{\lambda+\Gamma(A)}{2}, \\
& P-\limsup \sum_{m, n} \sum_{j}\left(a_{j k}^{m n}-v_{j k}\right)^{-} \leq \frac{\lambda-\Gamma(A)}{2},
\end{aligned}
$$

where for any $\gamma \in \mathbb{R}, \gamma^{+}=\max \{0, \gamma\}$ and $\gamma^{-}=\max \{-\gamma, 0\}$.

Proof. Since $A$ is RH-conservative, we have

$$
P-\limsup _{m, n} \sum_{j} \sum_{k}\left(a_{j k}^{m n}-v_{j k}\right)=\Gamma(A) .
$$

Therefore, the results follow from the relations

$$
\begin{aligned}
& \sum_{j} \sum_{k}\left(a_{j k}^{m n}-v_{j k}\right)=\sum_{j} \sum_{k}\left(a_{j k}^{m n}-v_{j k}\right)^{+}-\sum_{j} \sum_{k}\left(a_{j k}^{m n}-v_{j k}\right)^{-}, \\
& \sum_{j} \sum_{k}\left|a_{j k}^{m n}-v_{j k}\right|=\sum_{j} \sum_{k}\left(a_{j k}^{m n}-v_{j k}\right)^{+}+\sum_{j} \sum_{k}\left(a_{j k}^{m n}-v_{j k}\right)^{-} .
\end{aligned}
$$

Theorem 2.3. Let $A=\left[a_{j k}^{m n}\right]$ be RH-conservative. Then, for some constant $\lambda \geq|\Gamma(A)|$ and for all $x \in \ell_{\infty}^{2}$, one has

$$
P-\limsup \sum_{m, n} \sum_{k}\left(a_{j k}^{m n}-v_{j k}\right) x_{j k} \leq \frac{\lambda+\Gamma(A)}{2} L(x)-\frac{\lambda-\Gamma(A)}{2} l(x)
$$

if and only if (2.2) holds.

Proof. Suppose that (2.6) holds. Define the matrix $B=\left[b_{j k}^{m n}\right]$ by $b_{j k}^{m n}=\left(a_{j k}^{m n}-v_{j k}\right)$ for all $m, n, j, k \in \mathbb{N}$. Then, since $A$ is $\mathrm{RH}$-conservative, the matrix $B$ satisfies the hypothesis of Lemma 2.1. Hence, for a $y \in \ell_{\infty}^{2}$ such that $\|y\| \leq 1$, we have (2.1) with $b_{j k}^{m n}$ in place of $a_{j k}^{m n}$. So, from (2.6), we get that

$$
\begin{aligned}
P-\limsup _{m, n} \sum_{j} \sum_{k}\left|b_{j k}^{m n}\right| & \leq \frac{\lambda+\Gamma(A)}{2} L(y)-\frac{\lambda-\Gamma(A)}{2} l(y) \\
& \leq\left[\frac{\lambda+\Gamma(A)}{2}+\frac{\lambda-\Gamma(A)}{2}\right]\|y\| \leq \lambda
\end{aligned}
$$

which is (2.2). 
Conversely, suppose that (2.2) holds and $x \in \ell_{\infty}^{2}$. Then, for any $\varepsilon>0$, there exists an $N \in \mathbb{N}$ such that

$$
l(x)-\varepsilon<x_{j k}<L(x)+\varepsilon
$$

whenever $j, k>N$. Now, we can write

$$
\begin{aligned}
\sum_{j} \sum_{k} b_{j k}^{m n} x_{j k}= & \sum_{j \leq N} \sum_{k \leq N} b_{j k}^{m n} x_{j k}+\sum_{j \leq N} \sum_{k>N} b_{j k}^{m n} x_{j k}+\sum_{j>N} \sum_{k \leq N} b_{j k}^{m n} x_{j k} \\
& +\sum_{j>N} \sum_{k>N}\left(b_{j k}^{m n}\right)^{+} x_{j k}-\sum_{j>N} \sum_{k>N}\left(b_{j k}^{m n}\right)^{-} x_{j k},
\end{aligned}
$$

where $b_{j k}^{m n}$ is defined as above. Hence, by the RH-conservativeness of $A$ and Lemma 2.2, we obtain

$$
\begin{aligned}
P-\limsup _{m, n} \sum_{j} \sum_{k} b_{j k}^{m n} x_{j k} & \leq(L(x)+\varepsilon)\left(\frac{\lambda+\Gamma(A)}{2}\right)-(l(x)-\varepsilon)\left(\frac{\lambda-\Gamma(A)}{2}\right) \\
& =\frac{\lambda+\Gamma(A)}{2} L(x)-\frac{\lambda-\Gamma(A)}{2} l(x)+\lambda \varepsilon .
\end{aligned}
$$

Since $\varepsilon$ is arbitrary, this completes the proof.

In the case $\Gamma(A)>0$ and $\lambda=\Gamma(A)$, we have the following result.

Theorem 2.4. Let $A$ be RH-conservative and $x \in \ell_{\infty}^{2}$. Then,

$$
P-\limsup _{m, n} \sum_{j} \sum_{k}\left(a_{j k}^{m n}-v_{j k}\right) x_{j k} \leq \Gamma(A) L(x)
$$

if and only if

$$
P-\lim _{m, n} \sum_{j} \sum_{k}\left|a_{j k}^{m n}-v_{j k}\right|=\Gamma(A)
$$

Also, we should note that when $A$ is RH-regular, Theorem 2.4 is reduced to Lemma 1.1. Theorem 2.5. Let $A=\left[a_{j k}^{m n}\right]$ be RH-conservative. Then, for some constant $\lambda \geq|\Gamma(A)|$ and for all $x \in \ell_{\infty}^{2}$, one has

$$
P-\limsup \sum_{m, n} \sum_{j}\left(a_{j k}^{m n}-v_{j k}\right) x_{j k} \leq \frac{\lambda+\Gamma(A)}{2} L^{\text {ast }}(x)+\frac{\lambda-\Gamma(A)}{2} l^{\text {ast }}(-x)
$$

if and only if (2.2) holds and

$$
\begin{aligned}
& P-\lim _{m, n} \sum_{j} \sum_{k}\left|\Delta_{10} a_{j k}^{m n}\right|=0, \\
& P-\lim _{m, n} \sum_{j} \sum_{k}\left|\Delta_{01} a_{j k}^{m n}\right|=0,
\end{aligned}
$$


6 A class of conservative four-dimensional matrices

where

$$
\Delta_{10} a_{j k}^{m n}=a_{j k}^{m n}-a_{j+1, k}^{m n}-\left(v_{j k}-v_{j+1, k}\right), \quad \Delta_{01} a_{j k}^{m n}=a_{j k}^{m n}-a_{j, k+1}^{m n}-\left(v_{j k}-v_{j, k+1}\right) .
$$

Proof. Suppose that (2.13) holds. Then, since $L^{\text {ast }}(x) \leq L(x)$ and $l^{\text {ast }}(-x) \leq-l(x)$ for all $x \in \ell_{\infty}^{2}$ (see [3]), the necessity of (2.2) follows from Theorem 2.3.

Define a matrix $C=\left[c_{j k}^{m n}\right]$ by $c_{j k}^{m n}=\left(b_{j k}^{m n}-b_{j+1, k}^{m n}\right)$ for all $m, n, j, k \in \mathbb{N}$; where $b_{j k}^{m n}$ is as in Theorem 2.3. Then, we have from Lemma 2.1, a $y \in \ell_{\infty}^{2}$ such that $\|y\| \leq 1$ and (2.1) holds with $c_{j k}^{m n}$ in place of $a_{j k}^{m n}$. Also, for the same $y$, we can write

$$
\sum_{j} \sum_{k} c_{j k}^{m n} y_{j+1, k}=\sum_{j} \sum_{k} b_{j k}^{m n}\left(y_{j k}-y_{j+1, k}\right) .
$$

So, we have from $(2.13)$ that

$$
\begin{aligned}
P-\limsup _{m, n} \sum_{j} \sum_{k}\left|c_{j k}^{m n}\right| & =P-\limsup _{m, n} \sum_{j} \sum_{k} c_{j k}^{m n} y_{j+1, k} \\
& =P-\limsup _{m, n} \sum_{j} \sum_{k} b_{j k}^{m n}\left(y_{j k}-y_{j+1, k}\right) \\
& \leq \frac{\lambda+\Gamma(A)}{2} L^{\text {ast }}\left(y_{j k}-y_{j+1, k}\right)+\frac{\lambda-\Gamma(A)}{2} l^{\text {ast }}\left(y_{j+1}-y_{j k}\right) .
\end{aligned}
$$

Now, let $y=\left[y_{j k}\right]=1$ for all $j, k \in \mathbb{N}$. Then, since $\left(y_{j k}-y_{j+1, k}\right) \in f_{2}^{\infty, 0}$, the space of all double almost null sequences

$$
L^{\text {ast }}\left(y_{j k}-y_{j+1, k}\right)=l^{\text {ast }}\left(y_{j+1}-y_{j k}\right)=0 \text {. }
$$

This implies the necessity of (2.14). By the same argument one can prove the necessity of (2.15).

Conversely, suppose that the conditions (2.2), (2.14), and (2.15) hold. For any given $\varepsilon>0$, we can find integers $p, q \geq 2$ such that

$$
l^{\text {ast }}(-x)-\varepsilon<\frac{1}{p q} \sum_{j=m}^{m+p-1} \sum_{k=n}^{n+q-1} x_{j k}<L^{\text {ast }}(x)+\varepsilon
$$

whenever $j, k \geq N$. Now, one can write

$$
\sum_{j} \sum_{k} b_{j k}^{m n} x_{j k}=\sum_{1}+\sum_{2}+\sum_{3}+\sum_{4}
$$


where

$$
\begin{aligned}
& \sum_{1}=\sum_{j} \sum_{k} b_{j k}^{m n} \frac{1}{p q} \sum_{s=j}^{j+p-1} \sum_{t=k}^{k+q-1} x_{\mathrm{st}}, \\
& \sum_{2}=-\sum_{s=0} \sum_{t=0}^{p-2} \frac{1}{p q} \sum_{j=0}^{s} \sum_{k=0}^{t} b_{j k}^{m n} x_{\mathrm{st}}, \\
& \sum_{3}=-\sum_{j=p-1}^{\infty} \sum_{t=q-1}^{\infty}\left(\frac{1}{p q} \sum_{j=s-p+1}^{s} \sum_{k=t-q+1}^{t} b_{j k}^{m n}-b_{j k}^{m n}\right) x_{\mathrm{st}}, \\
& \sum_{4}=\sum_{j=0}^{p-2} \sum_{k=0}^{q-2} b_{j k}^{m n} x_{j k},
\end{aligned}
$$

and $b_{j k}^{m n}$ is defined as in Theorem 2.3. Then, since

$$
\left|\sum_{2}\right| \leq\|x\| \sum_{j=0}^{p-2} \sum_{k=0}^{q-2}\left|b_{j k}^{m n}\right|, \quad\left|\sum_{4}\right| \leq\|x\| \sum_{j=0}^{p-2} \sum_{k=0}^{q-2}\left|b_{j k}^{m n}\right|,
$$

using the condition (1.9), we observe that $\sum_{2} \rightarrow 0, \sum_{4} \rightarrow 0(m, n \rightarrow \infty)$. On the other hand, since

$$
\left|\sum_{3}\right| \leq \frac{\|x\|}{p q} \sum_{s=0}^{p-1} \sum_{t=0}^{q-1}\left((p-s-1) \sum_{j} \sum_{k}\left|\Delta_{10} a_{j k}^{m n}\right|+(q-t-1) \sum_{j} \sum_{k}\left|\Delta_{01} a_{j k}^{m n}\right|\right),
$$

by the conditions (2.14)-(2.15), $\sum_{3} \rightarrow 0(m, n \rightarrow \infty)$. Thus, we can write

$$
\begin{aligned}
\sum_{1}= & \sum_{j \leq N} \sum_{k \leq N} b_{j k}^{m n} \frac{1}{p q} \sum_{s=j}^{j+p-1} \sum_{t=k}^{k+q-1} x_{\mathrm{st}}+\sum_{j \geq N} \sum_{k \geq N} b_{j k}^{m n} \frac{1}{p q} \sum_{s=j}^{j+p-1} \sum_{t=k}^{k+q-1} x_{\mathrm{st}} \\
& -\sum_{j \geq N} \sum_{k \geq N} b_{j k}^{m n} \frac{1}{p q} \sum_{s=j}^{j+p-1} \sum_{t=k}^{k+q-1} x_{\mathrm{st}} .
\end{aligned}
$$

By (1.9), (2.20) and Lemma 2.2, we get that

$$
\begin{aligned}
P-\limsup _{m, n} \sum_{j} \sum_{k} b_{j k}^{m n} x_{j k} & \leq\left(L^{\text {ast }}(x)+\varepsilon\right) \frac{\lambda+\Gamma(A)}{2}+\left(l^{\text {ast }}(-x)+\varepsilon\right) \frac{\lambda-\Gamma(A)}{2} \\
& =\frac{\lambda+\Gamma(A)}{2} L^{\text {ast }}(x)+\frac{\lambda-\Gamma(A)}{2} l^{\text {ast }}(-x)+\lambda \varepsilon
\end{aligned}
$$

which is (2.13), since $\varepsilon$ is arbitrary.

In the case $\Gamma(A)>0$ and $\lambda=\Gamma(A)$, we have the following. 
8 A class of conservative four-dimensional matrices

Theorem 2.6. Let $A$ be $R H$-conservative and $x \in \ell_{\infty}^{2}$. Then,

$$
P-\limsup \sum_{m, n} \sum_{k}\left(a_{j k}^{m n}-v_{j k}\right) x_{j k} \leq \Gamma(A) L^{\text {ast }}(x)
$$

if and only if (2.12), (2.14), and (2.15) hold.

We should state that when $A$ is strongly regular, Theorem 2.6 is reduced to [3, Theorem 3.1].

\section{References}

[1] H. J. Hamilton, Transformations of multiple sequences, Duke Mathematical Journal 2 (1936), no. $1,29-60$.

[2] F. Móricz and B. E. Rhoades, Almost convergence of double sequences and strong regularity of summability matrices, Mathematical Proceedings of the Cambridge Philosophical Society 104 (1988), no. 2, 283-294.

[3] Mursaleen and O. H. H. Edely, Almost convergence and a core theorem for double sequences, Journal of Mathematical Analysis and Applications 293 (2004), no. 2, 532-540.

[4] R. F. Patterson, Double sequence core theorems, International Journal of Mathematics and Mathematical Sciences 22 (1999), no. 4, 785-793.

[5] A. Pringsheim, Zur Theorie der zweifach unendlichen Zahlenfolgen, Mathematische Annalen 53 (1900), no. 3, 289-321.

[6] G. M. Robison, Divergent double sequences and series, Transactions of the American Mathematical Society 28 (1926), no. 1, 50-73.

Celal Çakan: Faculty of Education, İnönü University, 44280 Malatya, Turkey

E-mail address: ccakan@inonu.edu.tr

Bilal Altay: Faculty of Education, İnönü University, 44280 Malatya, Turkey

E-mail address: baltay@inonu.edu.tr 